\title{
VCSELs for fast neuromorphic photonic systems operating at $\mathrm{GHz}$ rates
}

\author{
Matěj Hejda, Joshua Robertson, Julián Bueno \& Antonio Hurtado \\ Institute of Photonics, Dept. of Physics, University of Strathclyde, 99 George St., Glasgow, UK, G11RD \\ Authore-mail address: matej.hejda@strath.ac.uk
}

\begin{abstract}
We report experimentally on VCSEL-based artificial optical spiking neurons with ultrafast spiking refractory period; hence allowing operation at GHz rates. This feature is used to demonstrate all-optical digital-to-spiking information format conversion at $1.0 \mathrm{Gbps}$.
\end{abstract}

\section{Main Text}

Computational algorithms based on artificial neural networks are quickly becoming ubiquitous in our everyday lives. However, current computers, based on the von Neumann architecture, are not very well suited for the massively parallel nature of these algorithms. This inefficiency lead to the pursuit of new, "beyond von Neumann" hardware platforms, better suited for the tasks of the flourishing fields of artificial intelligence and computational neuroscience. The field of neuromorphic (brain-like) engineering answers those needs, mimicking the working architectures and principles of biological brains in large-scale networks of integrated circuits. Among the promising alternative technologies viable for neuromorphic platforms, photonics stands out due to the existence of low-loss optical interconnects, the intrinsic non-linear behaviour of photonic components, ultrafast operation rates, high power efficiency [1] and the possibility of wavelength-division multiplexing. Various photonic devices are being investigated as suitable neuromorphic systems, including optical modulators [2], resonant tunnelling diode-laser diode systems [3], quantum-dot lasers [4], semiconductor lasers [5] and micropillar lasers [6].

At the forefront of the emerging field of neuromorphic photonics are vertical cavity surface emitting lasers (VCSELs). These readily commercially available, compact, inexpensive devices, operating at standard telecom wavelengths, exhibit rich nonlinear dynamics governed by the same laws as biological neurons. We have previously shown that these VCSEL-neurons exhibit excitability, resulting in controlled excitation and inhibition of neuron-like spiking regimes [7-10] at speed rates up to 7 orders of magnitude higher than the time-scales of biological neurons. These excitable spikes are fundamentally hybrid in nature, being both analog (temporally continuous) and digital (binary in amplitude). Although the mystery of neural coding has not yet been fully understood, it is clear that precise spike timing is crucial for carrying information in biological neural networks [11]. Therefore, reliable, high-speed digital-to-spike convertors are essential to enable brain-like information encoding, as well as to interface these future neuromorphic computing platforms with conventional digital computers and telecommunication networks. In this work, we demonstrate that VCSELs exhibit one of the hallmark characteristics of biological neurons (the so-called absolute refractory period [12]) and experimentally determine the ultrafast temporal scale of this characteristic. Based on these findings, we then demonstrate experimentally an all-optical digital-to-spiking information format conversion system based on an artificial optical VCSEL-neuron.

\section{Experimental results}

The experimental setup of this work consisted of a tunable laser source whose light emission is optically-injected into a commercially available VCSEL operating at the important telecom wavelength of $1310 \mathrm{~nm}$. The latter acts as the artificial optical spiking neuron. The information input into the system is achieved by externally modulating the tunable laser's light intensity using a Mach-Zehnder modulator controlled by an arbitrary waveform generator. The light output from the VCSEL is collected and analysed with an optical spectrum analyser (Anritsu) and a $13 \mathrm{GHz}$ bandwidth real-time oscilloscope. For full details and a schematic depiction of the experimental setup see [13].

For the refractory period measurements, we used a waveform containing a sequence of fourteen square-shaped pulse pairs (pulse width $=160 \mathrm{ps}$ ) with a gradually increasing time separation between the pulses. The temporal separation $t$ is defined as the time between the rising pulse edges, and was inspected using temporal increments of 83 ps. Figs. $1(\mathrm{a}, \mathrm{b})$ show two different cases ( $t=916 \mathrm{ps}$ and $1166 \mathrm{ps}$, respectively) of multiple superimposed timetraces $(n=15)$ recorded at the output of the VCSEL-neuron using a $9.5 \mathrm{GHz}$ bandwidth photodetector connected to the oscilloscope. When the two input stimuli (pulse pair) are too close to each other (below the absolute refractory period, $t<\tau_{a b s}$ ), 
only one spike is fired by the VCSEL in response to the first stimulus. The second injected perturbation does not trigger an excitable response (Fig. 1(a)) due to depletion of gain carriers in the laser medium [14]. The two recorded small spike-like events in Fig. 1(a) can be attributed to signal jitter and pulse separation being very close to $\tau_{a b s}$. When the parameter $t$ is increased above the absolute refractory period $\left(t>\tau_{a b s}\right.$, Fig. 1(b)), two spiking events are successfully elicited in response to the two incoming perturbations (pulse pair). Fig. 1(c) shows the probability of second spiking event as a function of pulse pair temporal separation, $t$. There is a clear, sharp transition in measured probability occurring as the pulse separation $t$ approaches $1 \mathrm{~ns}$. Therefore, the absolute refractory period in our device for a given set of conditions is approximately equal to $\tau_{a b s} \cong 1 n s$, which is six orders of magnitude shorter than typical values for neurons in central nervous system [15]. It is important to mention that the exact value $\tau_{a b s}$ depends on multiple operating conditions of the system such as frequency detuning of the injected signal or applied bias on the VCSEL, as well as on the injection power and stimuli amplitude. Full details will be provided during the presentation.

\section{Evaluation of refractory period in VCSEL-neuron}
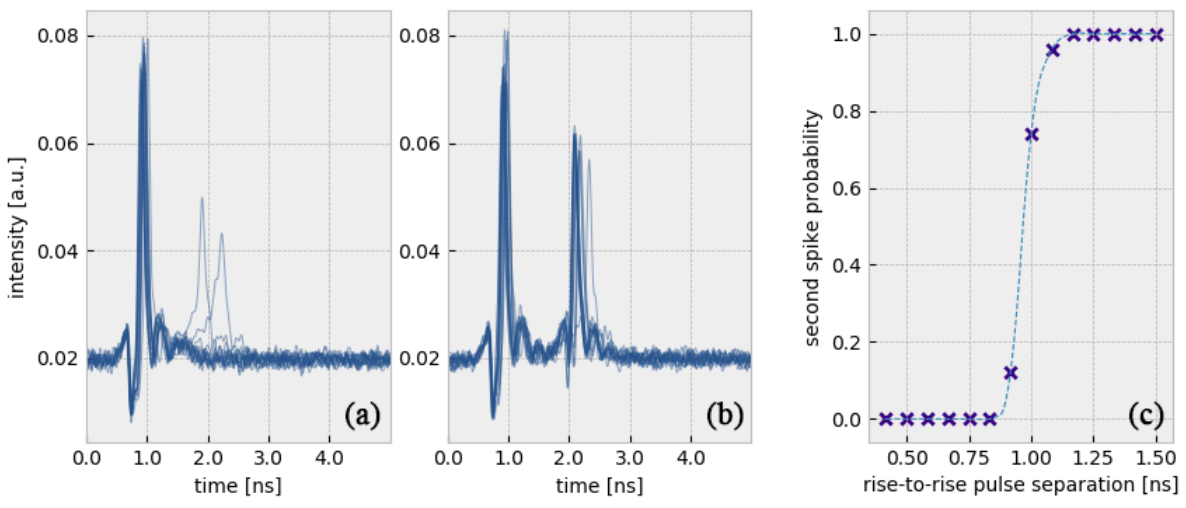

Fig. 1. (a,b) Overlay of VCSEL output intensity time-traces $(n=15)$ in response to incoming pulse pairs with different temporal separation between pulses. The first pulse (or stimulus) in both cases arrives when the VCSEL is in a rested state, and always triggers a spiking response. (a) The second pulse (stimulus) injected with a temporal separation (916 ps) right at the limit of the absolute refractory period does not trigger a second spike but only in a few small spike-like responses, while (b) for a temporal separation larger than the absolute refractory period (1160 ps) two spikes are fired by the VCSEL-neuron. (c) Probability of the VCSEL to fire a second spiking event versus temporal separation between the two spike-triggering perturbations, measured over a full ensemble of recorded cycles $(n=50)$.

In networks of biological neurons, the information signalled in spike trains depends either on the neuron firing rate (rate coding), on precise timing of individual spikes (temporal coding) or on the combination of both these mechanisms [16]. Hence, the ability to elicit spiking responses reliably and controllably is essential as a mean of injecting information into the neurons. Here, we introduce a method for all-optical digital-to-spike information format conversion at ultrafast rates using a VCSEL-based spiking optical neuron. The actual information is incorporated by externally modulating the intensity of the light from the tunable laser using the unipolar return-to-zero (RZ) serial encoding scheme. In this demonstration, the encoded information was an ASCII binary code representing the word "OFC" (24 bits of information, with the actual binary sequence highlighted above the plots in Fig. 2). In the spiking regime, the upper limit of the achievable spike frequency is defined by the previously introduced concept of absolute refractory period. Based on the previous findings, the temporal length of a single bit was selected as 1 ns (12 samples at $f=12 \mathrm{GHz}$ ) to match the absolute refractory period $\tau_{a b s}$, resulting in a coding rate of $1 \mathrm{Gbps}$. The duty cycle used in the RZ was $50 \%$, resulting in an input stimulus temporal length of $500 \mathrm{ps,} \mathrm{as} \mathrm{shown} \mathrm{on} \mathrm{Fig.} \mathrm{2(a).} \mathrm{An} \mathrm{overlay} \mathrm{of} \mathrm{five}$ subsequent cycles recorded at the output of the VCSEL-neuron is plotted in Fig. 2(b) showing a high degree of control over the spike activation in the system. Further analysis of all the responses recorded at the VCSEL-neuron's output revealed that during a single oscilloscope readout (120 cycles, 11 expected spikes in each, 1320 spikes in total), the desired single spike response (per non-zero bit) was elicited in all but one case, resulting in an accurate system's response in more than $99.9 \%$ cases. 


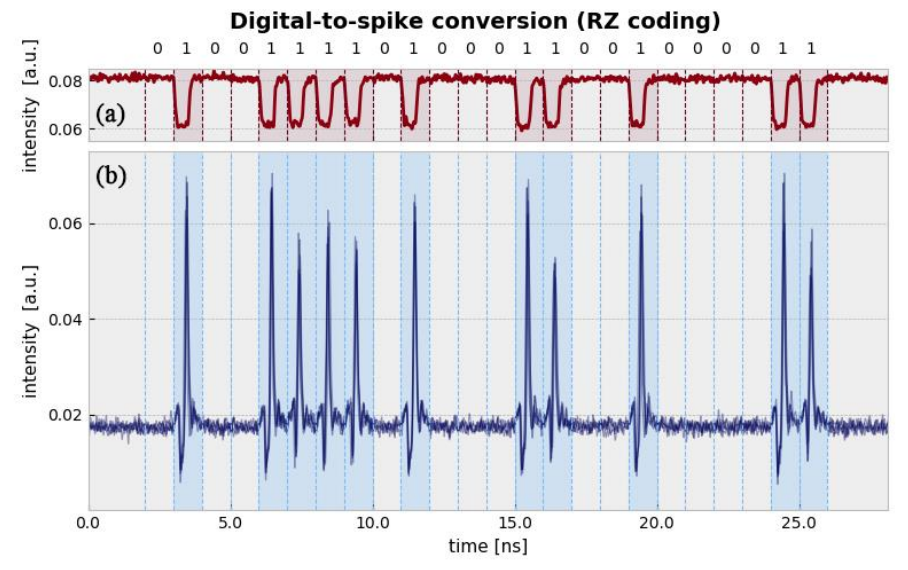

Fig. 2. All-optical digital-to-spiking information format conversion at $1 \mathrm{Gbps}$ with a VCSEL-neuron operating at $1310 \mathrm{~nm}$. The upper (red) time-trace shows the tunable laser's output with the applied intensity modulation that represents the desired information (word 'OFC' in ASCII binary code) in the form of bit-wise negative stimuli. The lower (blue) time-trace plots an overlay of multiple $(n=5)$ VCSEL-responses to this input signal. Regions of binary ' 1 ' (that are expected to elicit a spike) are highlighted in darker background colour.

\section{Conclusions}

In summary, we report experimentally on an artificial optical spiking neuron based on off-the-shelf, compact, commercially available VCSELs operating at standard telecom wavelengths. We show that this system exhibits refractoriness and we provide a measure of the absolute refractory period of $1 \mathrm{~ns}$ for the VCSEL-neuron of the work. This value is more than six orders of magnitudes faster than the time-scales of biological neurons. Utilizing the VCSEL-neuron's capability of producing precisely controllable ultrafast spiking patterns, we demonstrate in this work a highly reliable all-optical digital-to-spiking information format converter able to operate at 1 Gbps rates. Such a device forms an important link for interfacing between neuromorphic and traditional computers and further reinforces VCSELs as a promising platform for future neuromorphic photonic computational hardware implementations.

This work was supported by the Office of Naval Research Global (Grant ONRGNICOP-N62909-18-1-2027), the European Commission (Grant 828841-ChipAI-H2020-FETOPEN-2018-2020) and the EPSRC Doctoral Training Partnership (EP/N509760).

\section{References}

[1] D.A.B. Miller, "Attojoule Optoelectronics for Low-Energy Information Processing and Communications,” IEEE J. Light. Technol. 35(3), 346-396 (2017).

[2] A. N. Tait, et al., “A silicon photonic modulator neuron,” Phys. Rev. Applied, 11, 064043 (2019).

[3] B. Romeira, et al., "Excitability and optical pulse generation in semiconductor lasers driven by resonant tunnelling diode photo-detectors," Opt. Exp. 21(18), 20931-20940 (2013).

[4] C. Mesaritakis, et al., "Artificial Neuron Based on Integrated Semiconductor Quantum dot Mode-Locked Lasers," Sci. Rep. 6, 39317 (2016).

[5] P. R. Prucnal et al., "Recent progress in semiconductor excitable lasers for photonic spike processing," Adv. Opt. Photon. 8(2), 228-299 (2016).

[6] V. A. Pammi et al. "Photonic Computing With Single and Coupled Spiking Micropillar Lasers," IEEE J. Sel. Top. Quantum Electron. 26, 1500307 (2020).

[7] A. Hurtado \& J. Javaloyes, "Controllable spiking patterns in long-wavelength vertical cavity surface emitting lasers for neuromorphic photonics systems”, Appl. Phys. Lett. 107, 241103 (2015).

[8] J. Robertson et al., "Controlled inhibition of spiking dynamics in VCSELs for neuromorphic photonics: theory and experiments," Opt. Lett. 42(8), 1560-1563 (2017).

[9] T. Deng et al., "Controlled Propagation of Spiking Dynamics in Vertical-Cavity Surface-Emitting Lasers: Towards Neuromorphic Photonic Networks,” IEEE J. Sel. Top. Quantum Electron. 23(6), 1800408 (2017).

[10] J. Robertson et al., "Electrically Controlled Neuron-Like Spiking Regimes in Vertical-Cavity Surface-Emitting Lasers at Ultrafast Rates," IEEE J. Sel. Top. Quantum Electron. 25(6), 5100307 (2019).

[11] D.A. Butts et al., "Temporal precision in the neural code and the timescales of natural vision," Nature 449, 92-95 (2007).

[12] A.L. Hodgkin and A.F. Huxley, "A quantitative description of membrane current and its application to conduction and excitation in nerve," J. Physiol. 117(4), 500-544 (1952).

[13] J. Robertson et al., "Toward Neuromorphic Photonic Networks of Ultrafast Spiking Laser Neurons," IEEE J. Sel. Top. Quantum Electron. 26(1), 7700715 (2020).

[14] P. Y. Ma et al., "All-optical digital-to-spike conversion using a graphene excitable laser," Opt. Exp. 25(26), 33504-33513 (2017).

[15] F. Gabbiani \& S. J. Cox, "Quantification of Spike Train Variability," in Mathematics for Neuroscientists, $2^{\text {nd }}$ ed. (Academic Press, 2017).

[16] J. Huxter et al., "Independent rate and temporal coding in hippocampal pyramidal cells," Nature 425, 828-832 (2003). 